PROCEEDINGS OF THE

AMERICAN MATHEMATICAL SOCIETY

Volume 125, Number 12, December 1997, Pages 3509-3513

S $0002-9939(97) 04064-1$

\title{
FACTORIZATION OF AN INTEGRALLY CLOSED IDEAL IN TWO-DIMENSIONAL REGULAR LOCAL RINGS
}

\author{
MEE-KYOUNG KIM
}

(Communicated by Wolmer V. Vasconcelos)

\begin{abstract}
Let $(R, m, k)$ be a two-dimensional regular local ring with algebraically closed residue field $k$ and $I$ be an $m$-primary integrally closed ideal in $R$. Let $T(I)$ be the set of Rees valuations of $I$ and $k(v)$ be the residue field of the valuation ring $V$ associated with $v \in T(I)$. Assume that $(a, b)$ is any minimal reduction of $I$. We show that if $I$ is the product of the distinct simple $m$-primary integrally closed ideals in $(R, m, k)$, then $k(v)$ is generated by the image of $a / b$ over $k$ for all $v \in T(I)$, and the converse of this is also true.
\end{abstract}

\section{InTRODUCTION}

Throughout this paper $(R, m, k)$ will denote a 2-dimensional regular local ring (RLR for short) with residue field $k$ and quotient field $K$. Let $I$ be an $m$-primary integrally closed ideal in $(R, m, k)$. Concerning the structure of the integrally closed ideals in a 2-dimensional RLR $(R, m, k)$, O. Zariski proved three beautiful theorems which are the main background for this paper. Zariski's Product Theorem ([8], Appendix 5, Theorem $2^{\prime}$ ) says that any product of integrally closed ideals is integrally closed. Hence the set of Rees valuations of $I$ is

$$
T(I)=\bigcup_{\substack{a \neq 0 \\ a \in I}}\left\{v \mid v \text { is the valution of }(R[I / a])_{q}, q \in \operatorname{Min}(a R[I / a])\right\} .
$$

By Zariski's Unique Factorization Theorem ([8], Appendix 5, Theorem 3), I= $I_{1}^{\mu_{1}} \cdots I_{l}^{\mu_{l}}, \mu_{i} \geq 1$, where $I_{1}, \cdots, I_{l}$ are distinct simple $m$-primary integrally closed ideals in $(R, m, k)$. Zariski also set up an one-to-one correspondence between the set of simple integrally closed ideals of $R$ and the set of prime divisors of the second kind on $R$ ([8], Appendix 5, Theorem E). Therefore, if $\operatorname{Min}(m R[I / a])=\left\{q_{1}, \cdots, q_{\lambda}\right\}$ then $\lambda=l$ and, upon reordering, $v_{i}$ is the valuation of $(R[I / a])_{q_{i}}$, where $v_{i}$ is the prime divisor associated to the ideal $I_{i}$ for $i=1, \cdots, l$.

We denote by $k(v)$ the residue field of the valuation $\operatorname{ring} V$ associated with $v$. Assume that $k$ is an algebraically closed field. Then $k(v)$ is a simple transcendental field extension of $k$ for all $v \in T(I)$. Moreover, if $(a, b)$ is any minimal reduction of $I$, then for all $v \in T(I)$, the image of $a / b$ in $k(v)$ is transcendental over $k$. We show that if $I=I_{1} \cdots I_{l}$, where $I_{1}, \cdots, I_{l}$ are distinct simple $m$-primary integrally closed ideals in $(R, m, k)$, then $k(v)$ is generated by the image of $a / b$ over $k$ for

Received by the editors July 16, 1993 and, in revised form, July 12, 1996.

1991 Mathematics Subject Classification. Primary 13A18; Secondary 13B20, 13C05.

(C)1997 American Mathematical Society 
all $v \in T(I)$, and the converse of this is also true. Section 2 is devoted to some preliminaries. In section 3 , we will prove main results.

\section{Preliminaries}

Let $(A, n)$ be a local ring and $I$ an ideal of $A$. An ideal $J$ contained in $I$ is called a reduction of $I$ if $J I^{s}=I^{s+1}$ for some integer $s \geq 0$. A reduction $J$ of $I$ is called a minimal reduction of $I$ if $J$ is minimal with respect to being a reduction of $I$. The order $o(I)$ of an ideal $I$ of a local ring $(A, n)$ is $r$ if $I \subseteq n^{r}$ but $I \nsubseteq n^{r+1}$. We will use notation $e(I)$ to denote the multiplicity of an $n$-primary ideal $I$ of $A$. Recall that an ideal is simple if it is not the unit ideal and has no nontrivial factorization.

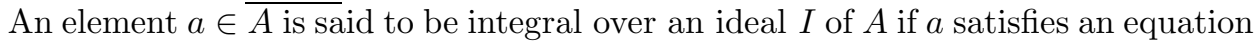
of the form

$$
a^{n}+r_{1} a^{n-1}+\cdots+r_{n}=0, \quad r_{i} \in I^{i} .
$$

The set of all elements in $A$ which are integral over an ideal $I$ forms an ideal, denoted by $\bar{I}$ and called the integral closure of $I$. An ideal $I$ is said to be integrally closed (or equivalently "complete") if $I=\bar{I}$.

In a $d$-dimensional local domain $(A, n, l)$ with quotient field $L$, by a prime divisor of the second kind on $\mathrm{A}$ (or equivalently prime divisor of $(A, n)$ ) we mean a discrete valuation $v$ of $L$ on $A$ which is non-negative on $A$ and has center $n$ on $A$ and whose residual transcendence degree (denoted by $\left.\operatorname{tr} \cdot \operatorname{deg}_{l} k(v)\right)$ is $d-1$.

Lemma 2.1. Let $(A, n)$ be a quasilocal normal domain with quotient field $L$. If $u \in L \backslash A$ is such that $u^{-1} \notin A$, then $n A[u]$ is a prime ideal in $A[u]$ and $A[u] / n A[u] \cong$ $(A / n)[X]$, a polynomial ring in one-variable over the field $A / n$.

Proof. Define the canonical homomorphism $\phi$ from $A[X]$ onto $A[u]$ with $\phi(X)=u$. $\operatorname{Ker}(\phi)$ is a prime ideal in $A[X]$ since $A[u]$ is a domain. By Theorem 11.13. in [7], $\operatorname{Ker}(\phi)$ is generated by linear polynomials $c X-d$ with $u=d / c$, where $c, d \in A$. It is not difficult to see that $c$ and $d$ are in the maximal idael $n$ of $A$. Hence we have the following exact sequence :

$$
0 \longrightarrow \frac{n A[X]}{\operatorname{Ker}(\phi)} \longrightarrow \frac{A[X]}{\operatorname{Ker}(\phi)} \stackrel{\bar{\phi}}{\longrightarrow} \frac{A[u]}{n A[u]} \longrightarrow 0,
$$

where $\bar{\phi}$ is the map induced by $\phi$. Hence we have $A[u] / n A[u] \cong A[X] / n A[X]$ is a polynomial ring one-variable over the field $A / n$, and so $n A[u]$ is a prime ideal in $A[u]$.

\section{MAIN RESUlts}

The following lemma will play a key role in the proofs of the first main result.

Lemma 3.1. Let $I$ be an m-primary integrally closed ideal in $(R, m, k)$. Assume that $\operatorname{Min}(m R[I t])=\left\{p_{1}, \cdots, p_{l}\right\}$ and that $k$ is an algebraically closed field. Let $v_{i}$ be the valuation of $R[I t]_{p_{i}} \cap K$ for $i=1, \cdots, l$. Then the following conditions are equivalent.

(1) $R[$ It $] / p_{i}$ is regular for $i=1, \cdots, l$.

(2) $I=I_{1} \cdots I_{l}$, where $I_{1}, \cdots, I_{l}$ are distinct simple $m$-primary integrally closed ideals in $R$. 
(3) For any reduction $(a, b)$ of $I$, there exist elements $c_{i_{1}}, \cdots, c_{i_{n}}$ in $I$ such that $a, b, c_{i_{1}}, \cdots, c_{i_{n}}$ is a minimal generating set of $I$ and $v_{i}\left(c_{i_{j}}\right)>v_{i}(a)=v_{i}(b)$ for $i=1, \cdots, l$ and $j=1, \cdots, n$.

(4) There exist a reduction $(a, b)$ of $I$ and elements $c_{i_{1}}, \cdots, c_{i_{n}}$ in $I$ such that $a, b, c_{i_{1}}, \cdots, c_{i_{n}}$ is a minimal generating set of $I$ and $v_{i}\left(c_{i_{j}}\right)>v_{i}(a)=v_{i}(b)$ for $i=1, \cdots, l$ and $j=1, \cdots, n$.

Proof. $(1) \Longleftrightarrow(2) \quad$ See $([5]$, Theorem 3.1).

$(1) \Longleftrightarrow(3) \Longleftrightarrow(4) \quad$ See $([5]$, Theorem 3.3).

Theorem 3.2. Let I be an m-primary integrally closed ideal in $(R, m, k)$ with an algebraically closed field $k$ and $(a, b)$ be any minimal reduction of $I$. Assume that $I=I_{1} \cdots I_{l}$, where $I_{1}, \cdots, I_{l}$ are distinct $m$-primary simple integrally closed ideals in $R$. Then $k(v)$ is generated by the image of $a / b$ over $k$ for all $v \in T(I)$.

Proof. Suppose that $I=I_{1} \cdots I_{l}$, where $I_{1}, \cdots, I_{l}$ are distinct simple $m$-primary integrally closed ideals in $R$. Let $v_{i}$ be a prime divisor of $R$ associated to $I_{i}$ for $i=1, \cdots, l$. Let $\operatorname{Min}(m R[I / b])=\left\{q_{1}, \cdots, q_{\lambda}\right\}$. By Zariski's One-to-One Correspondence Theorem, we have that $\lambda=l$ and, upon reordering, $v_{i}$ is the discrete valuation of $R[I / b]_{q_{i}}$ for $i=1, \cdots, l$, i.e., $T(I)=\left\{v_{1}, \cdots, v_{l}\right\}$. By Lemma 3.1, there exist elements $c_{i_{1}}, \cdots, c_{i_{n}}$ in $I$ such that $a, b, c_{i_{1}}, \cdots, c_{i_{n}}$ is a minimal generating set of $I$ and $v_{i}\left(c_{i_{j}}\right)>v_{i}(a)=v_{i}(b)$ for $i=1, \cdots, l$ and $j=1, \cdots, n$. For each $i=1, \cdots, l$, let $J_{i}=\left(m, c_{i_{1}} / b, \cdots, c_{i_{n}} / b\right)$. Then $J_{i} \subseteq q_{i}$ since $v_{i}\left(c_{i_{j}}\right)>v_{i}(a)=v_{i}(b)$ for $j=1, \cdots, n$. And we have

$$
\begin{aligned}
R[I / b] / J_{i} & =R[a / b] / m R[a / b] \\
& \cong(R / m)[X] \quad \text { by Lemma 3.1. }
\end{aligned}
$$

Let $(a / b)_{i}^{*}$ be the image of $a / b$ in $R[I / b] / J_{i}$ for $i=1, \cdots, l$. Since $J_{i}$ is a prime ideal in $R[I / b]$ and $\operatorname{dim}\left(R[I / b] / J_{i}\right)=\operatorname{dim}\left(R[I / b] / q_{i}\right)=1$, we have $J_{i}=q_{i}$ for $i=1, \cdots, l$. Thus $R[I / b] / q_{i}=(R / m)\left[(a / b)_{i}^{*}\right]$ for $i=1, \cdots, l$, which is a polynomial ring in one-variable over $k$. Localizing at $q_{i}$, we have $k\left(v_{i}\right)=(R / m)\left((a / b)_{i}^{*}\right)$ for $i=1, \cdots, l$.

Corollary 3.3 ([3], Remark 3.5). Let I be an m-primary integrally closed ideal in $(R, m, k)$ with an algebraically closed field $k$ and $(a, b)$ be any minimal reduction of I. Assume that $I$ is simple. Let $v$ be a prime divisor of $R$ associated to $I$. Then $k(v)$ is generated by the image of $a / b$ over $k$.

We remark that Theorem 3.2 does not extend, in general, to the case where $I$ is a power of a simple ideal.

Example 3.4. Let $R=k[x, y]_{(x, y)}$ and $m=(x, y) R$ with an algebraically closed field $k$. Let $I=m^{2}$. Then $T(I)=\{o\}$, where $o$ is the order valuation, i.e., the Rees valuation of $I$ is the $m$-adic prime divisor of $R, R[x / y]_{m R[x / y]} \cdot\left(x^{2}, y^{2}\right)$ is a minimal reduction of $I$. Since $m=(x, y)$ and $T(m)=\{o\}$, by Theorem 3.2, $k(o)=k(\theta)$, where $\theta$ is the image of $x / y$ in $R[x / y] / m R[x / y]$. Since $o\left(x^{2}\right)=o\left(y^{2}\right)=$ 2 , we have that $x^{2} / y^{2}$ is unit in $R[x / y]_{m R[x / y]}$, and hence the image of $x^{2} / y^{2}$ in $R[x / y] / m R[x / y]$ is $\theta^{2}$. But $k\left(\theta^{2}\right) \subseteq k(\theta)$.

Lemma 3.5 (Lipman, [2]). Let $I$ be an m-primary integrally closed ideal in $(R, m, k)$ with an algebraically closed field $k$. Assume that $I=I_{1}^{\mu_{1}} \cdots I_{l}^{\mu_{l}}$ is the 
unique factorization of I as a product simple integrally closed ideals $I_{1}, \cdots, I_{l}$. Then

$$
e(I)=\sum_{i=1}^{l} \mu_{i} v_{i}(I)
$$

where $v_{i}$ is the prime divisor associated to $I_{i}$ for $i=1, \cdots, l$.

Lemma 3.6 ([4], Theorem 1.1). Let $(A, n)$ be a local ring with infinite residue field and $J=\left(x_{1}, \cdots, x_{d}\right) A$ an ideal generated by a system of parameters of $A$. Set

$$
T=A\left[x_{1} / x_{d}, \cdots, x_{d-1} / x_{d}\right]_{n A\left[x_{1} / x_{d}, \cdots, x_{d-1} / x_{d}\right]} .
$$

Then $e(J)=e(J T)$.

Theorem 3.7. Let I be an m-primary integrally closed ideal in $(R, m, k)$ with an algebraically closed field $k$. Assume that $T(I)=\left\{v_{1}, \cdots, v_{l}\right\}$. Then the following conditions are equivalent.

(1) $I=I_{1} \cdots I_{l}$, where $I_{1}, \cdots, I_{l}$ are distinct simple m-primary integrally closed ideals in $R$.

(2) For any reduction $(a, b)$ of $I, k\left(v_{i}\right)=k\left((a / b)_{i}^{*}\right)$, where $(a / b)_{i}^{*}$ is the image of $a / b$ in $k\left(v_{i}\right)$ for $i=1, \cdots, l$.

(3) For some reduction $(a, b)$ of $I, k\left(v_{i}\right)=k\left((a / b)_{i}^{*}\right)$, where $(a / b)_{i}^{*}$ is the image of $a / b$ in $k\left(v_{i}\right)$ for $i=1, \cdots, l$.

Proof. (1) $\Rightarrow(2)$ : This follows immediately from Theorem 3.2.

$(2) \Rightarrow(3):$ It is clear.

$(3) \Rightarrow(1)$ : Assume that (3) is true. Let $J=(a, b)$. By Zariski's Unique Factorization and One-to-One Correspondence Theorems, $I=I_{1}^{\mu_{1}} \cdots I_{l}^{\mu_{l}}$, where $I_{1}, \cdots, I_{l}$ are distinct simple $m$-primary integrally closed ideals in $R$ and $\mu_{i} \geq 1$ for $i=1, \cdots, l$, and $\operatorname{Min}(m R[I / b])=\left\{q_{1}, \cdots, q_{l}\right\}$ and, upon reordering, $v_{i}$ is the discrete valuation of $R[I / b]_{q_{i}}$ for $i=1, \cdots, l . m R[a / b]$ is a prime ideal in $R[a / b]$ of $h t(m R[a / b])=1$ since $a, b$ are a regular sequence on $R$. Set $T=R[a / b]_{m R[a / b]}$. Then we have

$$
\begin{aligned}
e(I) & =\sum_{i=1}^{l} \mu_{i} v_{i}(I) \quad \text { by Lemma } 3.5 \\
& =e(J) \quad(J \text { is a reduction of } I) \\
& =e(J T) \quad \text { by Lemma } 3.6 .
\end{aligned}
$$

Let $T^{\prime}$ be the integral closure of $T$. Then $T^{\prime}=R[I / b]_{R[a / b]-m R[a / b]}$ is an onedimensional Noetherian normal domain since $R[a / b] \subseteq R[I / b]$ is integral and $R[I / b]$ is normal. By Corollary of the Krull-Akizuki Theorem ([6], Corollary of Theorem 11.7), there are just a finite number of maximal ideals of $T^{\prime}$ lying over $m T$.

Claim. $m R[a / b]=q_{i} \cap R[a / b]$ for $i=1, \cdots, l$.

Let $Q_{i}=q_{i} \cap R[a / b]$ for $i=1, \cdots, l$. Then $Q_{i} \supseteq m R[a / b]$. It is enough to show that $h t\left(Q_{i}\right)=1$ for $i=1, \cdots, l$. Suppose that $h t\left(Q_{i}\right)=2$. Then $R[a / b] / Q_{i} \cong$ $k[X] / Q_{i}^{\prime}$, where $Q_{i}^{\prime}$ is the image of $Q_{i}$ in $k[X]$, since $Q_{i} \supseteq m R[a / b]$. Hence the 0 -dimensional domain $R[a / b] / Q_{i}$ is a finitely generated $k$-algebra. By the Nullstellensatz ([1], Corollary 5.24), $R[a / b] / Q_{i}$ is a finite algebraic extension of $k$. Since $k$ is an algebraically closed field, $R[a / b] / Q_{i}=k$. But $(a / b)^{*} \in R[a / b] / Q_{i}$, where $(a / b)^{*}$ is the image of $a / b$ in $R[a / b] / Q_{i}$, which is a contradition since $(a / b)^{*}$ is transcendental over $k$. The proof of the claim is complete. 
By the above claim, $q_{1} T^{\prime}, \cdots, q_{l} T^{\prime}$ are all the maximal ideals of $T^{\prime}$ lying over $m T$ and

$$
T^{\prime}=\bigcap_{i=1}^{l}\left(T^{\prime}\right)_{q_{i} T^{\prime}}=\bigcap_{i=1}^{l} R[I / b]_{q_{i}} .
$$

By the projection formula ([8], Corollary 1, p. 299),

$$
e(J T)=\sum_{i=1}^{l}\left[T^{\prime} / q_{i} T^{\prime}: T / m T\right] e\left(J\left(T^{\prime}\right)_{q_{i} T^{\prime}}\right) .
$$

Since $R[a / b] / m R[a / b] \subseteq R[I / b] / q_{i}$ have the same quotient fields by the assumption, $T / m T=T^{\prime} / q_{i} T^{\prime}$ for all $i$. e $\left(J\left(T^{\prime}\right)_{q_{i} T^{\prime}}\right)=v_{i}(J)$ since $\left(T^{\prime}\right)_{q_{i} T^{\prime}}=R[I / b]_{q_{i}}$ is a Rees valuation ring of $I$ with associated valuation $v_{i}$ for $i=1, \cdots, l . v_{i}(J)=v_{i}(I)$ for all $i$ since $J \subseteq I$ is a reduction of $I$. We have

$$
e(J T)=\sum_{i=1}^{l} v_{i}(I)
$$

Hence we have

$$
\sum_{i=1}^{l} \mu_{i} v_{i}(I)=\sum_{i=1}^{l} v_{i}(I) .
$$

That is,

$$
\sum_{i=1}^{l}\left(\mu_{i}-1\right) v_{i}(I)=0
$$

Since $v_{i}(I)$ is positive value and $\mu_{i} \geq 1$ for all $i, \mu_{i}=1$ for all $i$. The proof is complete.

\section{REFERENCES}

1. M. Atiyah and I. MacDonald, Introduction to Commutative Algebra, Addison Wesley, 1969. MR 39:4129

2. C. Huneke, Lecture note on complete ideals in two-dimensional regular local rings, Purdue University, 1987.

3. _ and J. Sally, Birational extensions in dimension two and integrally closed ideals, J. of Algebra 115 (1988), 481-500. MR 89e:13025

4. D. Katz, Note on multiplicity, Proc. Amer. Math. Soc. 104-4 (1988), 1021-1026. MR 89d: 13017

5. M. K. Kim, Product of distinct simple integrally closed ideals in two dimensional regular local rings, Proc. Amer. Math. Soc. (to appear). CMP 96:14

6. H. Matsumura, Commutative ring theory, Cambridge Studies in Advanced Math. 8, Cambridge University Press, 1986. MR 88h:13001

7. M. Nagata, Local Rings, Interscience, New York, 1962. MR 27:5790

8. O. Zariski and P. Samuel, Commutative Algebra Vol.2., Van Nostrand, Princeton, 1960. MR 22:11006

Department of Mathematics, Sung Kyun Kwan University, Suwon 440 - 746, Korea

E-mail address: mkkim@yurim.skku.ac.kr 